\title{
Outcome and prognostic factors of multimodal therapy for pulmonary large-cell neuroendocrine carcinomas
}

\author{
Juliane Rieber ${ }^{1,2^{*}}$, Julian Schmitt ${ }^{1,2}$, Arne Warth $^{3}$, Thomas Muley ${ }^{4}$, Jutta Kappes ${ }^{4,5}$, Florian Eichhorn ${ }^{4,6}$, \\ Hans Hoffmann ${ }^{4,6}$, Claus Peter Heussel ${ }^{4,7,8}$, Thomas Welzel ${ }^{1,2}$, Jürgen Debus ${ }^{1,2}$, Michael Thomas ${ }^{4,5}$, \\ Martin Steins ${ }^{4,5+}$ and Stefan Rieken ${ }^{1,2 \dagger}$
}

\begin{abstract}
Background: There is controversy whether patients diagnosed with large-cell neuroendocrine carcinoma (LCNEC) should be treated according to protocols for non-small cell lung cancers (NSCLC) or small cell lung cancers (SCLC), especially with regard to the administration of prophylactic cranial irradiation $(\mathrm{PCl})$. This study was set up to determine the incidence of brain metastases and to investigate the outcome following multimodal treatment in 70 patients with LCNEC.

Methods: Seventy patients with histologically confirmed LCNEC were treated at the University Hospital of Heidelberg between 2001 and 2014. Data were collected retrospectively. Al most all patients received thoracic surgery as initial treatment (94\%). Chemotherapy was administered in 32 patients as part of the initial treatment. Fourteen patients were treated with adjuvant or definitive thoracic radiotherapy according to NSCLC protocols. Cranial radiotherapy due to brain metastases, mostly given as whole brain radiotherapy (WBRT), was received by fourteen patients. Statistical analysis was performed using the long-rank test and the Kaplan-Meier method.

Results: Without PCl, the detected rate for brain metastases was $25 \%$ after a median follow-up time of 23.4 months, which is comparable to NSCLC patients in general. Overall (OS), local (LPFS), brain metastases-free survival (BMFS) and extracranial distant progression-free survival (eDPFS) was 43,50,63 and $50 \%$ at 5 years, respectively. Patients with incomplete resection showed a survival benefit from adjuvant radiotherapy. The administration of adjuvant chemotherapy improved the general worse prognosis in higher pathologic stages.

Conclusion: In LCNEC patients, the administration of radiotherapy according to NSCLC guidelines appears reasonable and contributes to acceptable results of multimodal treatment regimes. The low incidence of spontaneous brain metastases questions a possible role of $\mathrm{PCl}$.
\end{abstract}

Keywords: Lung cancer, Large-cell neuroendocrine carcinoma, Radiotherapy, Brain metastases, Prophylactic cranial irradiation

\section{Background}

The incidence of large-cell neuroendocrine carcinoma (LCNEC) is low as it accounts for about $3 \%$ of all lung cancer cases $[1,2]$. Patients diagnosed with LCNEC

\footnotetext{
*Correspondence: juliane.rieber@med.uni-heidelberg.de

${ }^{+}$Martin Steins and Stefan Rieken contributed equally to this work

1 Department of Radiation Oncology, University Hospital Heidelberg, Im

Neuenheimer Feld 400, 69120 Heidelberg, Germany

Full list of author information is available at the end of the article
}

suffer from a very dismal prognosis with 5 -year overall survival rates between 15 and $57 \%$ [2-5]. During the last years, several reports suggested similarities in histology, clinical behavior and biology of LCNEC and small cell lung cancer (SCLC) [6-9]. Histological differentiation between LCNEC and SCLC can be challenging as both tumor entities often share many common features: neuroendocrine morphology, high mitotic rate, large zones of necrosis and positive immunohistochemical staining 
for neuroendocrine markers $[10,11]$. Furthermore, both LCNEC and SCLC are characterized by common clinical aspects including a predominance of males and smokers and aggressive clinical courses [11-13]. In addition, Jones et al. found comparable genetic alterations in LCNEC and SCLC and were unable to distinguish LCNEC from SCLC by gene expression profiling [14]. However, Ullmann et al. and Hiroshima et al. showed that LCNEC and SCLC harbor distinct morphological, phenotypical and genetical differences $[15,16]$. Moreover, analyzing 1,211 patients with LCNEC from the Surveillance, Epidemiology, and End Results (SEER) program of the US National Cancer Institute, Varlotto et al. reported that the clinical, histopathological and biological characteristics of LCNEC were more similar to large-cell carcinoma than to SCLC [17]. Additionally, the World Health Organization still categorizes LCNEC in the group of NSCLC.

Due to the complex clinical, histopathological and biological characteristics of LCNEC, it remains uncertain whether patients diagnosed with LCNEC should be treated according to NSCLC-based or SCLC-based regimes [11, 13, 17]. Current treatment strategies for patients with LCNEC are a mixture between guidelines for NSCLC and SCLC patients: while surgical resection is recommended for all non-metastatic stages analog to NSCLC treatment guidelines, adjuvant chemotherapy (when needed) is administered according to SCLC protocols $[13,18]$.

In general, treatment strategies also differ strongly between SCLC and NSCLC regarding radiotherapy. Patients diagnosed with SCLC with cN0 and pN1 nodal involvement (limited stage) are treated with thoracic radiotherapy, while patients with NSCLC only benefit from adjuvant radiotherapy in N2 nodal stages [19-21]. Prophylactic cranial irradiation (PCI) is recommended in patients with SCLC, as it prolongs both disease-free and overall survival $[22,23]$. On the contrary, $\mathrm{PCI}$ is not administered in patients with NSCLC, as Gore et al. only showed a decreased rate of brain metastases after PCI and were not able to detect any significant improvement of overall and disease-free survival after PCI in stage III lung cancer [24]. As treatment strategies vary strongly between SCLC and NSCLC, it is of major interest to find out more about the appropriate treatment regarding radiotherapy for patients with LCNEC.

\section{Methods}

Between 2001 and 2014, seventy patients with histologically confirmed large-cell neuroendocrine carcinoma of the lung were treated at the University Hospital in Heidelberg, Germany. This retrospective analysis was performed with ethical approval by the ethic committee of the University Hospital Heidelberg. Median follow-up time was 23 months (range 0-155 months). One patient was lost to follow-up after 18 months. Detailed patients' characteristics are shown in Table 1. For tumor grading and staging, the 7th lung cancer TNM classification was used $[25,26]$. In our cohort, most patients suffered from locally advanced stages (IIA-IIIB), while stage IV was only detected in seven patients.

Almost all patients underwent thoracic surgery as part of the initial treatment (93\%) (Table 1). According to current discussions and treatment recommendations, even some patients in oligometastatic tumor stage IV who were in good general health conditions and only suffered from few comorbidities received thoracic surgery

Table 1 Characteristics of 70 patients diagnosed with large-cell neuroendocrine carcinoma of the lung

\begin{tabular}{ll}
\hline Factor & LCNEC $(\boldsymbol{n}=\mathbf{7 0})$ \\
\hline Sex & \\
Male & $52(72 \%)$ \\
Female & $18(28 \%)$ \\
Median age (range) & 63 years (41-81) \\
Over 70 years & $20(29 \%)$ \\
Under 70 years or 70 years & $50(71 \%)$ \\
TNM stage (7th classification) & \\
IA/IB & $9 / 10$ \\
IIA/IIB & $15 / 9$ \\
IIIA/IIB & $18 / 2$ \\
IVA/IVB & 7 \\
Thoracic surgery & $66(94 \%)$ \\
Primary & $65(93 \%)$ \\
Wedge resection & $4(6 \%)$ \\
Lobectomy & $53(81 \%)$ \\
Bilobectomy & $3(5 \%)$ \\
Pneumonectomy & $5(8 \%)$ \\
Chemotherapy & $36(51 \%)$ \\
Definitive & $4(6 \%)$ \\
Adjuvant & $32(46 \%)$ \\
Relapse/progress & $14(20 \%)$ \\
Thoracic radiotherapy & $17(24 \%)$ \\
Definitive & $4(6 \%)$ \\
Adjuvant/additive & $10(14 \%)$ \\
Relapse/progress & $3(4 \%)$ \\
Resection & $17(24 \%)$ \\
Stereotactic irradiation & $7(10 \%)$ \\
Whole brain irradiation & $2(3 \%)$ \\
Proph supportive care & $6(9 \%)$ \\
\hline & $2(3 \%)$ \\
\hline & $3(4 \%)$ \\
\hline &
\end{tabular}


$[27,28]$. The discussion upon treatment in any case was taken by an interdisciplinary tumorboard conference.

Depending on comorbidity, age and cardiopulmonal function, chemotherapy was mostly administered postoperatively in stage IIA-IIIA according to the German S3-guideline [29]. The types of chemotherapeutic regimes were decided by the treating interdisciplinary team according to the current German S3-guideline at that time (Table 2).

Thirty-two patients received adjuvant chemotherapy after thoracic surgery. Of these patients, sixteen patients were treated according to SCLC-based regimes, while the further sixteen patients received chemotherapeutic treatment according to NSCLC-based regimes (Table 2). Stage IV patients without surgical resection were subjected to primary palliative (radio-) chemotherapy. Upon metastatic recurrence, seven patients received palliative chemotherapy.

Postoperative thoracic radiotherapy was administered in N2 stages and incomplete resections (R1 or R2). Doses with 50-60 Gy were used. Patients diagnosed with tumors at higher stages without surgical treatment options were treated with simultaneous radiochemotherapy administering photon doses of 66.0-70.5 Gy.

Two patients already suffered from brain metastases at the date of initial diagnosis, while seventeen patients developed brain metastases during follow-up time. All patients either received contrast-enhanced CT or MRI scan of the brain at initial diagnosis for staging. Before 2010 , only CT scans were performed, afterwards $48 \%$ of the patients were subjected to cranial staging with MRI. Treatment for brain metastases comprised whole brain radiotherapy, stereotactic irradiation and surgery. Three patients with locally advanced tumors received (PCI) with a total dose of $30.0 \mathrm{~Gy}$, single dose $2.0 \mathrm{~Gy}$ at initial diagnosis of LCNEC. Two of these patients received MRI Table 2 Different adjuvant chemotherapeutic regimes
used in treatment of patients with LCNEC

\begin{tabular}{lr}
\hline Chemotherapeutic regime & $\boldsymbol{N}$ \\
\hline SCLC-based regimes & \\
Cisplatin/etoposide & 11 \\
Carboplatin/etoposide & 5 \\
NSCLC-based regimes & 3 \\
Carboplatin/paclitaxel & 1 \\
Carboplatin/gemcitabine & 3 \\
Carboplatin/vinorelbine & 1 \\
Cisplatin/permetrexed & 5 \\
Cisplatin/vinorelbine & 2 \\
Cisplatin/doxetaxel & 1 \\
Gemcitabine & \\
\hline
\end{tabular}

scans, while one patient was staged using cranial CT due to claustrophobia.

\section{Statistical analysis}

Overall survival (OS) was calculated in months from the date of initial diagnosis until the last date of follow-up or death. Progression-free survival (PFS), local progression-free survival (LPFS), brain metastases-free survival (BMFS) and extracranial distant progression-free survival (DPFS), as well as brain metastases-free survival, were calculated from the date of primary diagnosis until the first imaging diagnosis of recurrent disease. Death due to any cause or lost to follow-up (one patient) was taken as an event. Data analysis was censored as not all patients suffered from an event during follow-up time. The Kaplan-Meier method was used to display results. Survival curves were compared between groups in a univariate analysis using the long-rank test. All statistical analyses were performed with a software tool (SPSS 20.0).

\section{Results}

The follow-up period for the patients in this study ranged from 0 months to 155 months. The median follow-up time was 23 months. The survival curve for the seventy patients with LCNEC is shown in Fig. 1a. The 2-year and the 5-year overall survival rates (OS) were 74 and $43 \%$, respectively. Survival was dependent on tumor stage: patients with stage I-II LCNEC showed 2- and 5-year overall survival rates of 67 and $48 \%$, while patients diagnosed with stage III-IV LCNEC had a 2- and 5-year overall survival of 39 and $29 \%$. 2- and 5-year progression-free survival rates (PFS) were 74 and $38 \%$, respectively (Fig. 1b). Local progression-free survival was $75 \%$ after 2 years and $50 \%$ after 5 years (Fig. 1c). 2 - and 5 -year extracranial distant progression-free survival was 53 and $50 \%$, respectively (Fig. 1d).

\section{Brain metastases}

Seventeen patients (25\%) were diagnosed with brain metastases during follow-up. While 9 patients only suffered from 1 to 2 brain metastases, 8 patients presented with disseminated metastases (more than three). Patients presenting with 1-2 metastases either received surgical resection or radiosurgery. Hence, brain metastases-free survival was $85 \%$ after 2 years and $63 \%$ after 5 years (Fig. 2a). Brain metastases-free survival was not influenced by age and sex. Interestingly, the development of brain metastases was significantly associated with pathologic stage when comparing stage I vs. stage II-IV tumors $(p=0.045$; Fig. 2b).

Patients with brain metastases suffered from significantly reduced overall survival with 2- and 5-year rates 

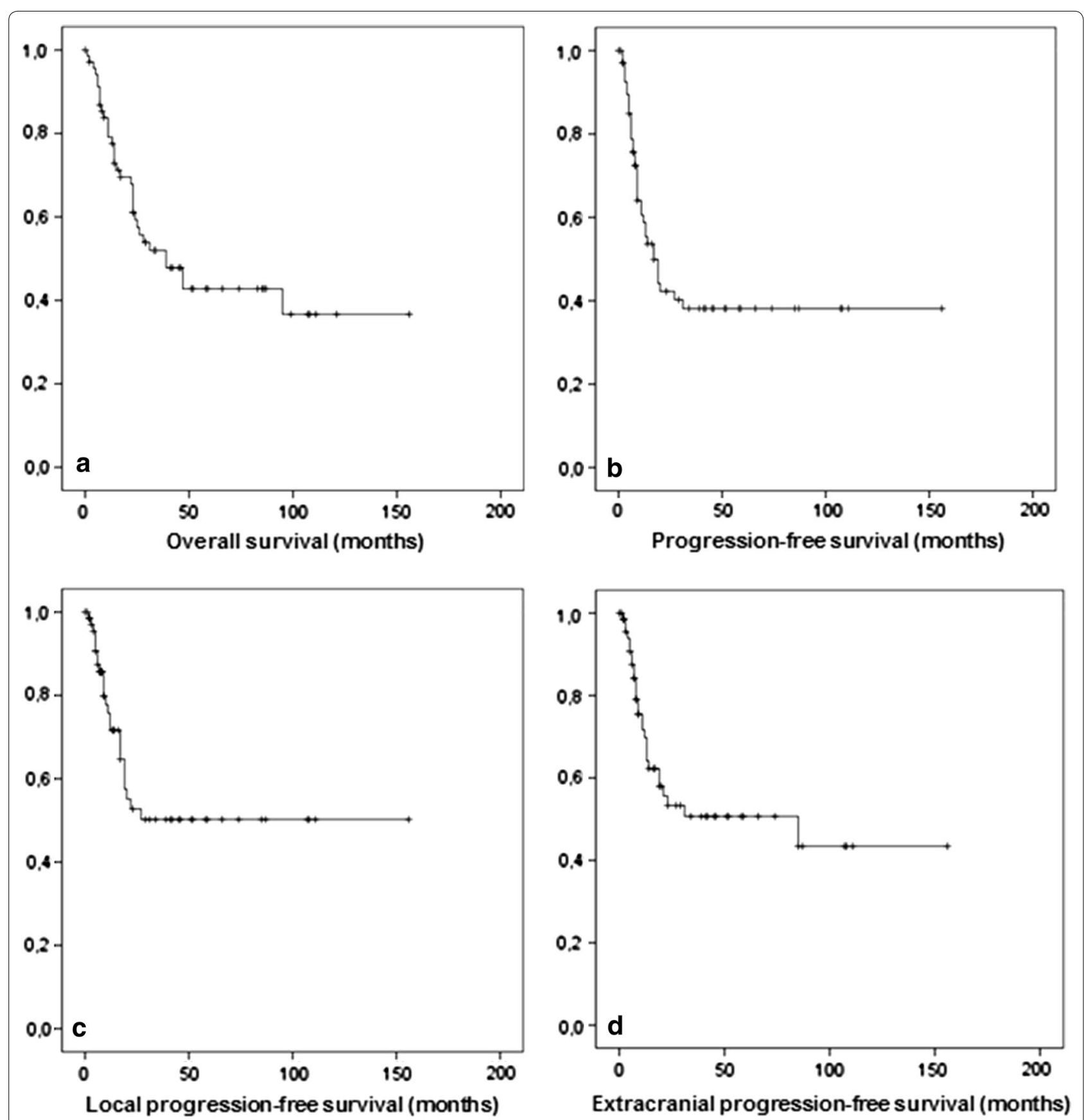

Fig. 1 Overall (a) and progression-free (b), local (c) and extracranial distant progression-free survival (d) in 70 patients with LCNEC.

of 37 and $16 \%$, while patients without brain metastases showed 2- and 5-year overall survival rates of 69 and $54 \%$, respectively $(p=0.002)$. Along with the introduction of SCLC-derived chemotherapy regimes, three patients with advanced tumor stages received prophylactic cranial irradiation (PCI). Patients with PCI did not develop brain metastases during follow-up time (8.2 months). During follow-up time, 37 (53 \%) LCNEC patients developed progression with local relapse in 25 patients (36\%) and distant progression in 28 patients (40\%). First relapse was distant in 6 (16\%) and combined local and distant at the same time in 31 patients (83.4\%).

Apart from the patients with cerebral metastases, 17 patients (24\%) developed distant metastases: liver (7), bone (7), adrenal (4) and lung (2). 


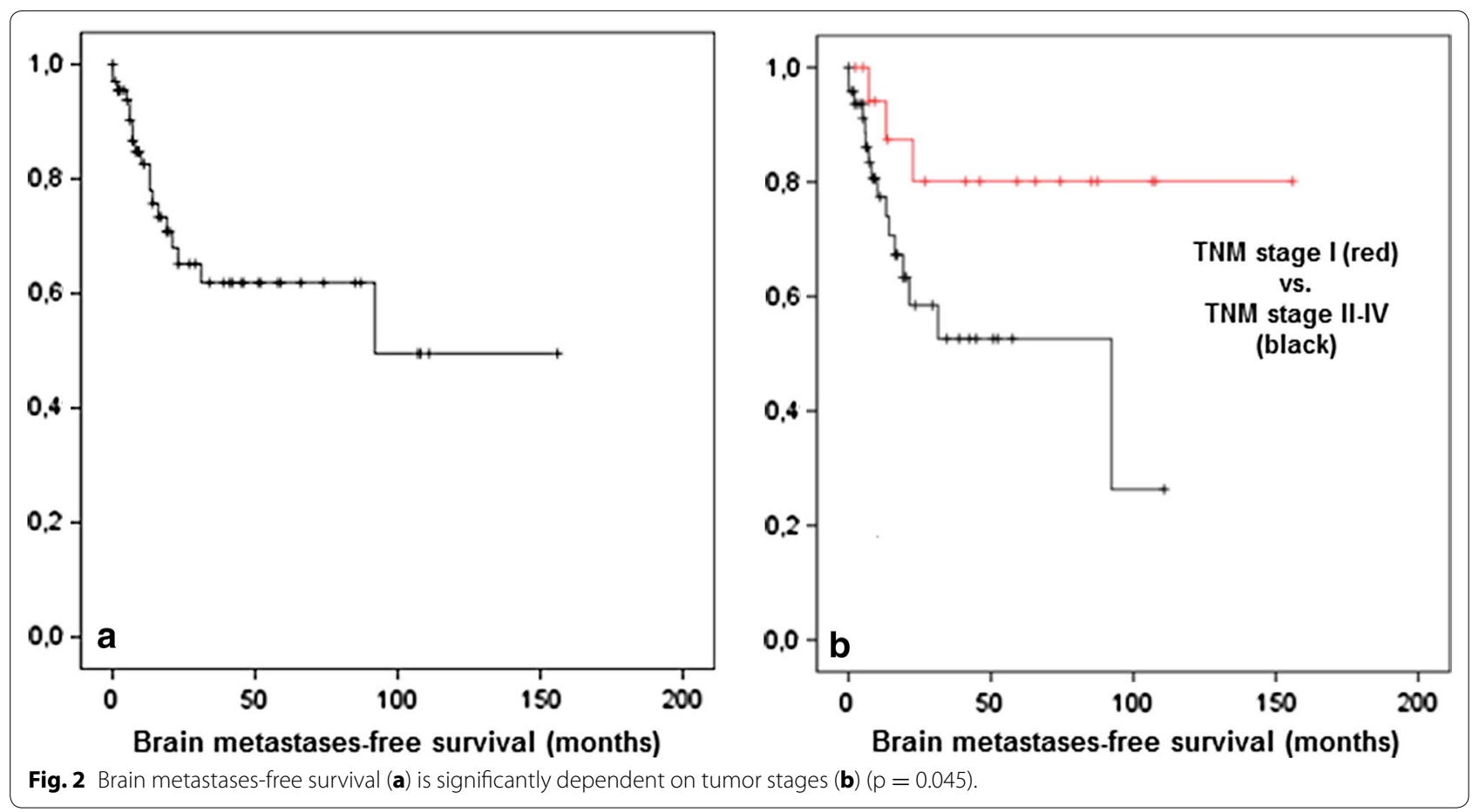

\section{Treatment concepts}

Patients with incompletely resected LCNEC tumors received postoperative radiotherapy and showed noninferior survival rates with 2- and 5-year overall survival of 50 and $30 \%$, respectively ( $p=0.89$ ). In addition, we analyzed the different treatment concepts regarding overall and disease-free survival. Chemotherapy was administered in heterogeneous regimes (Table 2). According to the German S3-guideline, patients in higher stages were treated with adjuvant chemo-, radio- or radiochemotherapy after primary resection [29]. Regarding overall survival, we compared patients who only received resection as primary treatment $(n=34 ; 53 \%)$ to the patients who were treated with resection and adjuvant chemo-, radioor radiochemotherapy, mainly because of higher tumor stages ( $\geq$ IIIA) $(n=30 ; 47 \%)$. Both groups did not differ in overall survival ( $p=0.298$ ) (Fig. 3). Additionally, local progression-free survival was also not significantly different between these two groups $(p=0.412)$. Furthermore, comparing chemotherapy regimes derived from SCLC vs. NSCLC protocols, we did not detect a difference in treatment results and patient outcome.

\section{Discussion}

In the present study, we evaluated incidence of brain metastases, treatment outcome and prognostic factors in 70 patients with $\mathrm{LCNEC}$ who received multimodal treatment. In our cohort, 17 patients (25\%) were diagnosed with brain metastases during follow-up of 2 years.

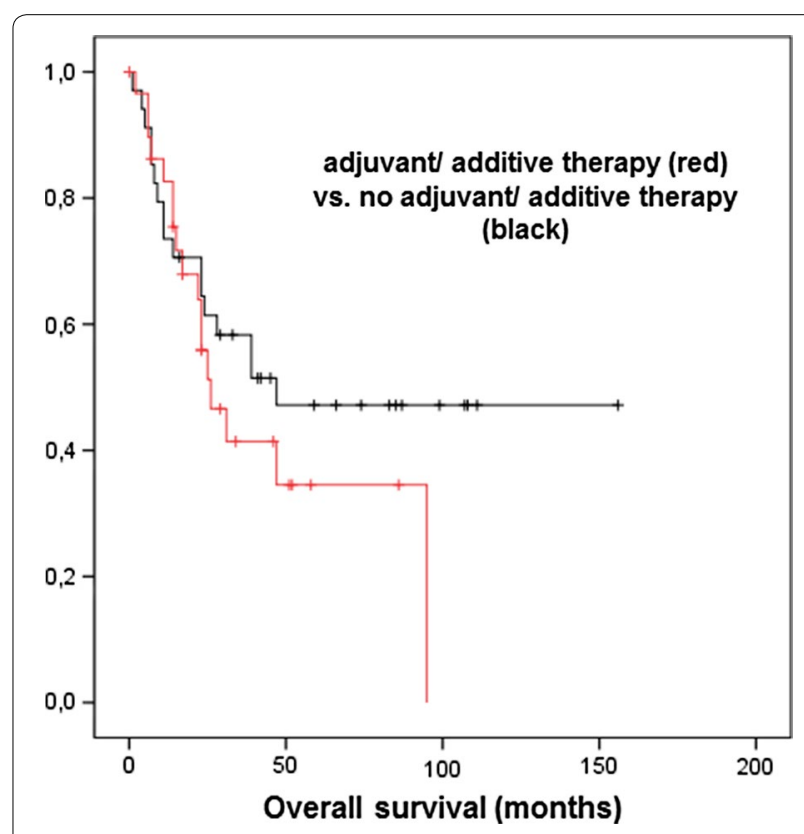

Fig. 3 Adjuvant chemotherapy in higher pathologic stages improves overall survival.

In NSCLC, $20-30 \%$ of patients are diagnosed with brain metastases, while in SCLC already $50 \%$ of patients suffer from brain metastases 2 years after initial diagnosis $[22,30,31]$. Due to the complex clinicopathological and biological features of patients with LCNEC, there is no 
consensus on whether LCNEC should be treated according to SCLC or NSCLC protocols [13]. Among others, Sun et al. claimed that LCNEC should receive treatment similar to SCLC, while Varlotto et al. insisted on treating LCNEC with NSCLC treatment regimes [11, 17, 32]. As Aupérin et al. showed that prophylactic cranial irradiation (PCI) improves both overall and disease-free survival among patients with limited disease SCLC in complete remission, $\mathrm{PCI}$ became standard treatment in patients with limited disease SCLC [22, 29]. Some years later, Slotman et al. established PCI for patients with extensive disease SCLC [23]. Regarding patients diagnosed with NSCLC, prophylactic cranial irradiation is usually not recommended in higher pathologic stages. Gore et al. only detected a decreased rate of brain metastases after PCI and were not able to show any significant improvement of overall or disease-free survival after PCI in stage III lung cancer [24, 29]. Nevertheless, Iyoda et al. proposed that PCI might be promising for patients with LCNEC [33]. Indeed, the three patients who received PCI in our cohort did not develop brain metastases during follow-up time (8.23 months). However, in our study $25 \%$ of patients developed brain metastases during follow-up which is comparable to patients with NSCLC and not to patients with SCLC. Similar to our results, Iyoda et al. showed that about $19 \%$ of the analyzed LCNEC patients developed brain metastases [32, 34]. Interestingly, the development of brain metastases was significantly associated with pathologic TNM stage in our cohort. We detected a continuous, non-saturable but shallow decline in brain metastases-free survival. In general, patients with SCLC in lower stages tend to show the greatest benefit from PCI [22]. As on the one hand, only few LCNEC patients develop brain metastases and on the other hand, there was a strong correlation between pathologic stage and the development of brain metastases in our study, prophylactic cranial irradiation (PCI) in patients with LCNEC should be reconsidered thoroughly, especially in lower tumors stages.

LCNEC used to be only treated by resection in all tumor stages and therefore showed poor survival rates $[1,35,36]$. Adjuvant therapy, mainly chemotherapy, led to a subsequent improvement in survival in patients with higher tumor stages [5, 37, 38]. In NSCLC, adjuvant thoracic radiotherapy is only recommended in $\mathrm{pN} 2$ and higher nodal stages and after incomplete resection. On the contrary, patients with SCLC benefit from thoracic radiotherapy when diagnosed with $\mathrm{cN} 0$ and $\mathrm{pN} 1$ nodal involvement (limited stage) [19-21, 29]. LCNEC tumors appear to be radiation responsive as patients with incompletely resected LCNEC and adjuvant radiotherapy showed non-inferior survival. However, excellent local control was detected in patients with limited thoracic tumor extension. Hence, radiotherapy is not needed in lower tumor stages and should only be applied according to NSCLC treatment protocols.

Sarkaria et al. reported that LCNEC had a high response rate to platinum-based chemotherapy. Furthermore, resected advanced stage patients receiving combination neoadjuvant and (or) adjuvant chemotherapy tended to have a survival benefit [39]. Correspondently, additional treatment with adjuvant or additive chemo-, radio-, or radiochemotherapy in higher stage large-cell neuroendocrine carcinoma led to overall survival rates comparable to patients who underwent surgery only for low-stage NSCLC. This fact underlines the importance of adjuvant treatment in LCNEC regarding overall survival. Sun et al. revealed that the response rate to platinum-based chemotherapy was $60 \%$ in LCNEC patients, whereas the response rate of non-platinumbased chemotherapy was only $11 \%$ [11]. Interestingly, all but one patient in our cohort received adjuvant platinum-based chemotherapy which might have led to the improved survival rates. Yamazaki et al. also reported a similar response rate to platinum-based chemotherapy and suggested that the response rate of LCNEC patients was comparable to that of SCLC patients [40]. Due to the good response rate to platinum-based chemotherapy and the partly comparable histopathological features of LCNEC and SCLC, mainly chemotherapeutic regimes used in the treatment of SCLC were evaluated within the last years [10].

In our cohort, treatment results and patient outcome was not significantly different comparing chemotherapy regimes derived from SCLC versus NSCLC patients. This may be related to platinum administration in both SCLC and NSCLC regimes in our cohort (Table 2). Rossi et al. analyzed 83 patients with LCNEC and showed that SCLC-based chemotherapy (platinum-etoposide) was an important variable significantly correlating with survival, both in adjuvant and metastatic settings [41]. Iyoda et al. started a prospective trial of adjuvant chemotherapy with platinum and etoposide and reported that SCLC-based chemotherapy appeared promising for the improvement of overall survival in LCNEC patients [42]. Similarly, Treut et al. conducted a multicenter phase II study and found that the outcomes of advanced LCNEC treated with cisplatin and etoposide were comparable to that of advanced SCLC [43]. Recently, a phase III trial comparing irinotecan and cisplatin with etoposide and cisplatin in adjuvant chemotherapy for completely resected LCNEC was initiated [44].

\section{Conclusion}

Despite poor survival rates, only $25 \%$ of LCNEC patients developed brain metastases and therefore, PCI should 
not be considered a standard component of LCNEC treatment regimes. Adjuvant or additive therapy according to the current German S3-guideline in higher stage large-cell neuroendocrine carcinoma led to overall survival rates comparable to patients who underwent surgery only for low-stage NSCLC, putting emphasis on the need for most effective first-line treatments. Despite its rare occurrence, prospective multi-center trials are needed to truly evaluate the optimal multi-modal therapy for patients with LCNEC.

\section{Authors' contributions}

JR carried out the data collection, statistical analysis and drafted the manuscript, JS, AW and TM provided data and assisted with data analysis. JK and TM were responsible for systemic treatment of the patients, while FE and $\mathrm{HH}$ performed surgical resection of the analyzed patients. $\mathrm{CPH}$ and TW did radiological diagnosis. JD was responsible for radiotherapy treatment. MS and SR conceived of the study, and participated in its design and coordination and helped to draft the manuscript. All authors read and approved the final manuscript.

\section{Author details \\ ${ }^{1}$ Department of Radiation Oncology, University Hospital Heidelberg, Im Neuenheimer Feld 400, 69120 Heidelberg, Germany. ${ }^{2}$ Heidelberg Institute of Radiation Oncology, Heidelberg, Germany. ${ }^{3}$ Institute for Pathology, University Hospital Heidelberg, Im Neuenheimer Feld 224, 69120 Heidelberg, Germany. ${ }^{4}$ Translational Research Unit, Translational Lung Research Centre Heidelberg (TLRC-H), German Centre for Lung Research (DZL), Im Neuenhe- imer Feld 430, 69120 Heidelberg, Germany. ${ }^{5}$ Department of Thoracic Oncol- ogy, Thoraxklinik, Heidelberg University, Amalienstraße 20, 69126 Heidelberg, Germany. ${ }^{6}$ Department of Thoracic Surgery, Thoraxklinik, Heidelberg Univer- sity, Amalienstraße 20, 69126 Heidelberg, Germany. ${ }^{7}$ Department of Diag- nostic and Interventional Radiology with Nuclear Medicine, Thoraxklinik gGmbH, University Hospital Heidelberg, Heidelberg, Germany. ${ }^{8}$ Department of Diagnostic and Interventional Radiology, University Hospital Heidelberg, Heidelberg, Germany.}

\section{Acknowledgements}

We acknowledge financial support by Deutsche Forschungsgemeinschaft and Ruprecht-Karls-Universität Heidelberg within the funding programme Open Access Publishing.

This work was supported by the Medical Faculty of Heidelberg University providing a research grant for JR.

\section{Compliance with ethical guidelines}

\section{Competing interests}

The authors declare that they have no competing interests.

Received: 26 May 2015 Accepted: 3 August 2015

Published online: 14 August 2015

\section{References}

1. Jiang SX, Kameya T, Shoji M, Dobashi Y, Shinada J, Yoshimura H (1998) Large cell neuroendocrine carcinoma of the lung: a histologic and immunohistochemical study of 22 cases. Am J Surg Pathol 22:526-537

2. Takei H, Asamura H, Maeshima A, Suzuki K, Kondo H, Niki T et al (2002) Large cell neuroendocrine carcinoma of the lung: a clinicopathologic study of eighty-seven cases. J Thorac Cardiovasc Surg 124:285-292

3. Battafarano RJ, Fernandez FG, Ritter J, Meyers BF, Guthrie TJ, Cooper JD et al (2005) Large cell neuroendocrine carcinoma: an aggressive form of non-small cell lung cancer. J Thorac Cardiovasc Surg 130:166-172

4. Kinoshita T, Yoshida J, Ishii G, Aokage K, Hishida T, Nagai K (2013) The differences of biological behavior based on the clinicopathological data between resectable large-cell neuroendocrine carcinoma and small-cell lung carcinoma. Clin Lung Cancer 14:535-540

5. Veronesi G, Morandi U, Alloisio M, Terzi A, Cardillo G, Filosso P et al (2006) Large cell neuroendocrine carcinoma of the lung: a retrospective analysis of 144 surgical cases. Lung Cancer 53:111-115

6. Asamura H (2011) Identity, similarity, and difference between large cell neuroendocrine carcinoma and small cell carcinoma. J Thorac Oncol 6:1774 (author reply 1776)

7. Iyoda A, Baba M, Hiroshima K, Saitoh H, Moriya Y, Shibuya Ket al (2004) Imprint cytologic features of pulmonary large cell neuroendocrine carcinoma: comparison with classic large cell carcinoma. Oncol Rep 11:285-288

8. Sun L, Sakurai S, Sano T, Hironaka M, Kawashima O, Nakajima T (2009) High-grade neuroendocrine carcinoma of the lung: comparative clinicopathological study of large cell neuroendocrine carcinoma and small cell lung carcinoma. Pathol Int 59:522-529

9. Eichhorn F, Dienemann H, Muley T, Warth A, Hoffmann H (2015) Predictors of survival after operation among patients with large cell neuroendocrine carcinoma of the lung. Ann Thorac Surg 99:983-989

10. Igawa S, Watanabe R, Ito I, Murakami H, Takahashi T, Nakamura Y et al (2010) Comparison of chemotherapy for unresectable pulmonary highgrade non-small cell neuroendocrine carcinoma and small-cell lung cancer. Lung Cancer 68:438-445

11. Sun J-M, Ahn M-J, Ahn JS, Um S-W, Kim H, Kim HK et al (2012) Chemotherapy for pulmonary large cell neuroendocrine carcinoma: similar to that for small cell lung cancer or non-small cell lung cancer? Lung Cancer 77:365-370

12. Gollard R, Jhatakia S, Elliott M, Kosty M (2010) Large cell/neuroendocrine carcinoma. Lung Cancer 69:13-18

13. Iyoda A, Makino T, Koezuka S, Otsuka H, Hata Y (2014) Treatment options for patients with large cell neuroendocrine carcinoma of the lung. Gen Thorac Cardiovasc Surg 62:351-356

14. Jones MH, Virtanen C, Honjoh D, Miyoshi T, Satoh Y, Okumura S et al (2004) Two prognostically significant subtypes of high-grade lung neuroendocrine tumours independent of small-cell and large-cell neuroendocrine carcinomas identified by gene expression profiles. Lancet 363:775-781

15. Hiroshima K, lyoda A, Shida T, Shibuya K, lizasa T, Kishi H et al (2006) Distinction of pulmonary large cell neuroendocrine carcinoma from small cell lung carcinoma: a morphological, immunohistochemical, and molecular analysis. Mod Pathol 19:1358-1368

16. Ullmann R, Petzmann S, Sharma A, Cagle PT, Popper HH (2001) Chromosomal aberrations in a series of large-cell neuroendocrine carcinomas: unexpected divergence from small-cell carcinoma of the lung. Hum Pathol 32:1059-1063

17. Varlotto JM, Medford-Davis LN, Recht A, Flickinger JC, Schaefer E, Zander DS et al (2011) Should large cell neuroendocrine lung carcinoma be classified and treated as a small cell lung cancer or with other large cell carcinomas? J Thorac Oncol 6:1050-1058

18. Gridelli C, Rossi A, Airoma G, Bianco R, Costanzo R, Daniele B et al (2013) Treatment of pulmonary neuroendocrine tumours: state of the art and future developments. Cancer Treat Rev 39:466-472

19. (1998) Postoperative radiotherapy in non-small-cell lung cancer: systematic review and meta-analysis of individual patient data from nine randomised controlled trials. Lancet 352:257-263

20. Douillard J-Y, Rosell R, De Lena M, Riggi M, Hurteloup P, Mahe M-A (2008) Impact of postoperative radiation therapy on survival in patients with complete resection and stage I, II, or IIIA non-small-cell lung cancer treated with adjuvant chemotherapy: The Adjuvant Navelbine International Trialist Association (ANITA) Randomized Trial. Int J Radiat Oncol Biol Phys 72:695-701

21. Pignon J-P, Arriagada R, Ihde DC, Johnson DH, Perry MC, Souhami RL et al (1992) A meta-analysis of thoracic radiotherapy for small-cell lung cancer. N Engl J Med 327:1618-1624

22. Auperin A, Arriagada R, Pignon JP, Le Pechoux C, Gregor A, Stephens RJ et al (1999) Prophylactic cranial irradiation for patients with small-cell lung cancer in complete remission. Prophylactic Cranial Irradiation Overview Collaborative Group. N Engl J Med 341:476-484

23. Slotman B, Faivre-Finn C, Kramer G, Rankin E, Snee M, Hatton M et al (2007) Prophylactic cranial irradiation in extensive small-cell lung cancer. N Engl J Med 357:664-672 
24. Gore EM, Bae K, Wong SJ, Sun A, Bonner JA, Schild SE et al (2011) Phase III comparison of prophylactic cranial irradiation versus observation in patients with locally advanced non-small-cell lung cancer: primary analysis of radiation therapy oncology group study RTOG 0214. J Clin Oncol 29:272-278

25. Goldstraw P, Crowley J, Chansky K, Giroux DJ, Groome PA, Rami-Porta Ret al (2007) The IASLC Lung Cancer Staging Project: proposals for the revision of the TNM stage groupings in the forthcoming (seventh) edition of the TNM classification of malignant tumours. JThorac Oncol 2:706-714

26. Groome PA, Bolejack V, Crowley JJ, Kennedy C, Krasnik M, Sobin LH et al (2007) The IASLC Lung Cancer Staging Project: validation of the proposals for revision of the T, N, and $\mathrm{M}$ descriptors and consequent stage groupings in the forthcoming (seventh) edition of the TNM classification of malignant tumours. J Thorac Oncol 2:694-705

27. Ashworth A, Rodrigues G, Boldt G, Palma D (2013) Is there an oligometastatic state in non-small cell lung cancer? A systematic review of the literature. Lung Cancer 82:197-203

28. Ashworth AB, Senan S, Palma DA, Riquet M, Ahn YC, Ricardi U et al (2014) An individual patient data metaanalysis of outcomes and prognostic factors after treatment of oligometastatic non-small-cell lung cancer. Clin Lung Cancer 15:346-355

29. Goeckenjan G, Sitter H, Thomas M, Branscheid D, Flentje M, Griesinger F et al (2011) Prevention, diagnosis, therapy, and follow-up of lung cancer: interdisciplinary guideline of the German Respiratory Society and the German Cancer Society. Pneumologie 65:39-59

30. Gerdan L, Segedin B, Nagy V, Khoa MT, Trang NT, Schild SE et al (2014) Brain metastasis from non-small cell lung cancer (NSCLC). Strahlenther Onkol 190:64-67

31. Zabel A, Debus J (2004) Treatment of brain metastases from nonsmall-cell lung cancer (NSCLC): radiotherapy. Lung Cancer 45(Suppl 2):S247-S252

32. Iyoda A, Hiroshima K, Moriya Y, Iwadate Y, Takiguchi Y, Uno T et al (2009) Postoperative recurrence and the role of adjuvant chemotherapy in patients with pulmonary large-cell neuroendocrine carcinoma. J Thorac Cardiovasc Surg 138:446-453

33. Iyoda A, Hiroshima K, Nakatani Y, Fujisawa T (2007) Pulmonary large cell neuroendocrine carcinoma: its place in the spectrum of pulmonary carcinoma. Ann Thorac Surg 84:702-707
34. Iyoda A, Jiang SX, Travis WD, Kurouzu N, Ogawa F, Amano H et al (2013) Clinicopathological features and the impact of the new TNM classification of malignant tumors in patients with pulmonary large cell neuroendocrine carcinoma. Mol Clin Oncol 1:437-443

35. Dresler CM, Ritter JH, Patterson GA, Ross E, Bailey MS, Wick MR (1997) Clinical-pathologic analysis of 40 patients with large cell neuroendocrine carcinoma of the lung. Ann Thorac Surg 63:180-185

36. Travis WD, Colby TV, Corrin B, Shimosato Y, Brambilla E (1999) Histological typing of lung and pleural tumors, 3 rd edn. Springer, Berlin

37. Iyoda A, Hiroshima K, Toyozaki T, Haga Y, Baba M, Fujisawa T et al (2001) Adjuvant chemotherapy for large cell carcinoma with neuroendocrine features. Cancer 92:1108-1112

38. Kozuki T, Fujimoto N, Ueoka H, Kiura K, Fujiwara K, Shiomi K et al (2005) Complexity in the treatment of pulmonary large cell neuroendocrine carcinoma. J Cancer Res Clin Oncol 131:147-151

39. Sarkaria IS, lyoda A, Roh MS, Sica G, Kuk D, Sima CS et al (2011) Neoadjuvant and adjuvant chemotherapy in resected pulmonary large cell neuroendocrine carcinomas: a single institution experience. Ann Thorac Surg 92:1180-1187

40. Yamazaki S, Sekine I, Matsuno Y, Takei H, Yamamoto N, Kunitoh H et al (2005) Clinical responses of large cell neuroendocrine carcinoma of the lung to cisplatin-based chemotherapy. Lung Cancer 49:217-223

41. Rossi G, Cavazza A, Marchioni A, Longo L, Migaldi M, Sartori G et al (2005) Role of chemotherapy and the receptor tyrosine kinases KIT, PDGFRalpha, PDGFRbeta, and Met in large-cell neuroendocrine carcinoma of the lung. J Clin Oncol 23:8774-8785

42. Iyoda A, Hiroshima K, Moriya Y, Takiguchi Y, Sekine Y, Shibuya K et al (2006) Prospective study of adjuvant chemotherapy for pulmonary large cell neuroendocrine carcinoma. Ann Thorac Surg 82:1802-1807

43. Le Treut J, Sault MC, Lena H, Souquet PJ, Vergnenegre A, Le Caer H et al (2013) Multicentre phase II study of cisplatin-etoposide chemotherapy for advanced large-cell neuroendocrine lung carcinoma: the GFPC 0302 study. Ann Oncol 24:1548-1552

44. Eba J, Kenmotsu H, Tsuboi M, Niho S, Katayama H, Shibata T et al (2014) A phase III trial comparing irinotecan and cisplatin with etoposide and cisplatin in adjuvant chemotherapy for completely resected pulmonary high-grade neuroendocrine carcinoma (JCOG1205/1206). Jpn J Clin Oncol 44:379-382

\section{Submit your next manuscript to BioMed Central and take full advantage of:}

- Convenient online submission

- Thorough peer review

- No space constraints or color figure charges

- Immediate publication on acceptance

- Inclusion in PubMed, CAS, Scopus and Google Scholar

- Research which is freely available for redistribution

Submit your manuscript at

www.biomedcentral.com/submit

C BioMed Central 